\title{
GAUSSIAN FITTING PARAMETERS OF THE ESA PLANCK HFI BEAMS
} \author{
Jean-Michel Lamarre, ${ }^{2}$ and Julien Brossard ${ }^{3,4}$ \\ ${ }^{1}$ Experimental Physics Department \\ National University of Ireland \\ Maynooth, Co. Kildre, Ireland \\ ${ }^{2}$ Observatoire de Paris \\ 61 Av de l'Observatoire \\ 75014, Paris, France \\ ${ }^{3}$ CNES, Centre Spatial de Toulouse \\ 18, av Edouard Belin \\ 31401 Toulouse, France \\ ${ }^{4}$ CESR, 9 Av du Colonel Roche \\ 31401, Toulouse, France
}

Vladimir B. Yurchenko, ${ }^{1}$ John Anthony Murphy,

Received February 9, 2004

\begin{abstract}
$\underline{\text { Abstract }}$
We present Gaussian fitting parameters of simulated beams of the High Frequency Instrument (HFI) of the ESA PLANCK mission. This space probe is designed for measuring the anisotropy of temperature and polarization of the Cosmic Microwave Background (CMB). The six HFI spectral bands cover the frequency range $0.1-1 \mathrm{THz}$ with 52 bolometers. Their beams are computed by multi-mode physical optics propagation of the source field from the apertures of the horns simulated by the scattering matrix approach. Computed power patterns are fitted by the elliptical Gaussian beams minimizing the peak difference between the two power distributions within the beam. This approximation is generally considered as acceptable from the scientific viewpoint, although we show that induced errors are far from negligible.
\end{abstract}

Key words: PLANCK mission, CMB, THz PO simulations 


\section{Introduction}

The ESA PLANCK mission is the $3^{\text {rd }}$ generation deep-space satellite being designed for measuring the temperature anisotropies and polarization of the cosmic microwave background (CMB). The satellite will carry a dual-reflector multi-beam telescope equipped with two focal plane instruments, the Low-Frequency and High-Frequency Instruments, for detecting the radiation in the frequency range from $30 \mathrm{GHz}$ to 1000 $\mathrm{GHz}[1]$ in nine bands.

The High-Frequency Instrument (HFI) [2] will operate in six frequency channels centred at $100,143,217,353,545$ and $857 \mathrm{GHz}$ It is equipped with 36 corrugated horn antennas feeding cryogenically cooled bolometric detectors. Channels operating at $100,143,217$ and $353 \mathrm{GHz}$ use diffraction-limited mono-mode quasi-Gaussian horns [3]. Half of them will be used for polarization measurements by utilizing polarizationsensitive micromesh bolometers developed by Turner et al. [4].

The higher frequency channels (545 and $857 \mathrm{GHz}$ ) are non-polarized and incorporate non-diffraction-limited profiled multi-mode horns. All the HFI horns are broad band, with the bandwidth being about $30 \%$ of central frequency. The horns are designed to meet the extreme requirements on both the primary mirror edge taper $(\sim 25-30 \mathrm{~dB})$ and the angular resolution on the sky (about 5 arcminutes at the frequencies of $217-857 \mathrm{GHz}$ ).

Since both the temperature anisotropy $\varepsilon=\delta T / T$ and the degree of polarization $d$ of the CMB radiation are extremely small $\left(\varepsilon \sim 10^{-4}\right.$ and $d<$ $10^{-5}$ ), the $\mathrm{CMB}$ measurements require an exquisite accuracy of the instrument and a tight control of systematic errors. To achieve this goal, thorough simulations and testing of the instrument are needed.

The horns are distributed in the focal plane over several degrees (see Table 1). Due to the position of all horns and to the very compact design of the off-axis dual mirror telescope, the beam shapes are far from circular. Their exact representation requires mapping with a sinificant number of points (at least $128 \times 128$ ). This becomes important because the simulation and data reduction of the Planck mission will be huge tasks requiring enormous computing power. Especially, convolving or deconvolving a whole sky high resolution image with complex beams represented by at least thousands of points makes the operation impracticable. A first step towards feasibility is to model the beams by analytical functions that can be handled more efficiently than the raw data [5]. 
The aim of this paper is to provide a wide community with a first set of data that can be used as a reference for simulations of the Planck missions and for the development of its data processing tools. In the same time, it describes tools that can be used in other projects. In the next section, we describe the method and summarise the results of our simulations of the beams. In section 3 , we describe in detail the fitting parameters and methodology. The fourth section is dedicated to the presentation of the results and a short discussion of their quality.

\section{Simulation of the PLANCK HFI beams}

Simulations of the PLANCK HFI beams are extremely challenging because of the dual-reflector geometry of the telescope, large primary mirror having projected diameter $D=1.5 \mathrm{~m}\left(D / \lambda_{\min } \sim 5000\right)$, strict requirements on the accuracy and multi-mode structure of the feed horn antenna fields. Physical optics (PO) is the most appropriate technique for this kind of simulations. Conventional software cannot, however, cope efficiently with the problem of this size in the full PO simulation mode.

To overcome this limitattion, we developed a dedicated ultra-fast PO code $[6,7]$ that allowed us to perform rigorous PO+PO TE/TM-mode simulations of the main beams (typically, on the sky mesh of $128 \times 128$ nodes, with the convergence accuracy better than $0.1 \%$ of the beam maximum power) in tens of minutes for mono-mode channels at a single frequency and in a few hours for the broad-band polarization-averaged multi-mode channels using laptop PC Dell Latitude C840 under the Linux operating system.

We compute the beam patterns of the IQUV Stokes parameters in the far field of the ESA PLANCK telescope by propagating the source field from the apertures of the actual CQM horns via the telescope mode-bymode, with integration over the frequency bands and with account of all polarization directions of the horn field of non-polarized channels.

The electric field at the aperture of the profiled corrugated horns has been computed by the scattering matrix approach [8]. The effective modes of the electric field at the horn aperture, $\boldsymbol{E}_{n m}$, are represented via the canonical TE-TM modes $\varepsilon_{n j}$ of a cylindrical waveguide as follows

$$
\boldsymbol{E}_{n m}(\rho, \varphi)=\Sigma_{j=1, \ldots, 2 M} S_{n m j} \varepsilon_{n j}(\rho, \varphi)
$$

where $S_{n m j}=S_{n m j}(f)$ is the scattering matrix provided by E. Gleeson [8] for each CQM horn at various frequencies $f\left(S_{n m j}\right.$ is used as an input in 
this work), $n=0,1, \ldots, N$ is the azimuthal index and $m, j=1,2, \ldots, 2 M$ are the radial indices accounting for both the TE $(m, j=1, \ldots, M)$ and TM $(m, j=M+1, \ldots, 2 M)$ modes.

In multi-mode beams, both the power contribution and the shape of the $\boldsymbol{E}_{n m}$ modes vary significantly within the bandwidth, with many modes being rejected at the lower frequency edge (Fig. 1, a). The total power of the horn field is the sum of powers of the $\boldsymbol{E}_{n m}$ modes with account of all polarization directions. Similarly, the beam patterns of Stokes parameter are the sums of the relevant patterns of all the modes at all the frequencies of the bandwidth. In mono-mode beams, all the $\boldsymbol{E}_{n m}$ modes are of the same shape and effectively sum up to a single mode which is of one unit of total power, almost Gaussian in shape and, for the polarized channels, of nearly perfect linear polarization on the horn aperture. Broad-band and mono-frequency far-field power patterns of, e.g., polarized HFI-143 horns are shown in Fig. 1, b.

In this work, the HFI beams are computed assuming smooth telescope mirrors of ideal elliptical shape, of perfect electrical conductivity of their reflective surfaces, and of ideal positioning of mirrors and horn antennas. The horn positions are specified by the aperture refocus parameter $R_{A}=$ $R_{F}+R_{C}$ where $R_{A}$ is the distance along the horn axis from the reference detector plane to the horn aperture, $R_{F}$ is the similar distance to the point $\mathrm{F}$ of the geometrical focus of telescope on this axis, and $R_{C}$ is the distance from the point $\mathrm{F}$ to the horn aperture.

The best refocus $R_{A O}$ is found by minimizing the angular width of the broad-band beams (simultaneously, it appears to maximize the gain, even in the case of complicated multi-mode beams). For the design reasons, however, the actual refocus of some horns (e.g., HFI-353 and 857) is slightly different from $R_{A 0}$. At the best refocus, the value $R_{C O}=R_{A 0}-R_{F}$

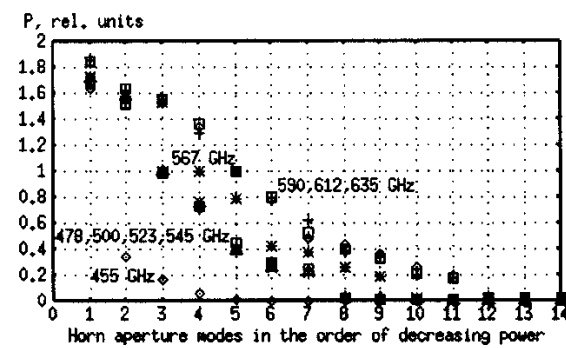

(a)

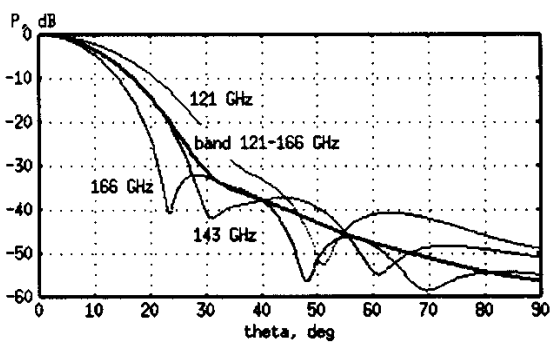

(b)

Fig. 1: Contribution of $\boldsymbol{E}_{n m}$ modes to HFI-545 beams (all polarizations) in units of power of a single polarized mode; (b) power patterns of the HFI-143 horns 
specifies the focal center of the horn. This is the point inside the horn which is superimposed with the telescope focal point $\mathrm{F}$ when $R_{A}=R_{A 0}$.

The beam data below are computed assuming $R_{C}=0.5,1.3,1.6,1.5$, 4.5 and $1.0 \mathrm{~mm}$ for the frequency channels HFI-100, 143, 217, 353, 545 and 857 , respectively. Here, in some cases, $R_{C}=R_{C O}$ (e.g., for the polarized beams HFI-100, 143, 217 and multi-mode HFI-545), otherwise, $R_{C}<R_{C O}$. The actual design values may slightly differ from these numbers.

Measuring polarization with bolometric detectors requires comparisons of signals of four polarization channels obtained from the same pixel on the sky. To minimize polarization errors due to mismatch of different beams, pairs of orthogonal polarization channels $(a, b)$ are built into each horn by using polarization-sensitive bolometers (PSB) [4]. In this case, the difference of power patterns of two channels of the same beam is, typically, about $1 \%$ of maximum power [9]. It arises only due to minor asymmetry of the polarized modes on the horn aperture (caused by the field propagation through the horn) and a slight difference in the propagation of different polarizations along the same path via the telescope. The two other measurements needed to evaluate the $Q$ and $U$ Stokes parameters are obtained less than a second later, when the sister beam observes the same pixel, due to the spinning of the satellite at 1 RPM and the layout of the horns in the focal plane. The mismatch of sister beams when superimposed on the sky by spinning the telescope is much greater (up to 5-8\%) and depends on the horn location in the focal plane [9].

In all our simulations, we assume ideal PSBs with no cross-polarization leakage. In absence of a better model of the PSB, more realistic IQUV patterns can be estimated from the available $I Q U V$ data as follows

$$
\boldsymbol{I}_{a}=c_{a a} I_{a}+c_{a b} I_{b}, \quad \boldsymbol{I}_{b}=c_{b a} I_{a}+c_{b b} I_{b}
$$

and, similarly, $Q U V$ where $c_{a b}$ is the matrix representing the PSB a/b polarization leakage due to electronics cross-talk, extra mode mixing in the PSB, some PSB non-orthogonality in the horns, etc.

\section{Fitting parameters of the HFI beams}

In this paper, we use the HFI beams computed at the central frequency of each channel, except for the multi-mode beams HFI-545 and HFI-857. The multi-mode beams have a complicated structure which depends 
essentially on the frequency within the channel. So, they are computed as broad-band, using 9 sampling frequencies and up to 11 modes in the band $f=455-635 \mathrm{GHz}$ for the HFI-545 beams (see Fig. 1, a) and, similarly, 3 representative frequencies $f=716,857,998 \mathrm{GHz}$ with up to 16 modes in the band $f=716-998 \mathrm{GHz}$ for the HFI- 857 beams. The broad-band IQUV patterns in the latter case are computed as, e.g., $I=I_{857}+0.5\left(I_{716}+I_{998}\right)$ where $I_{f}$ is the relevant Stokes parameter at the frequency $f$.

The IQUV beam patterns are presented as functions of the $\left(\varphi_{S C}, \eta_{S C}\right)$ coordinates on the sky $\left(\eta_{S C}=90^{\circ}-\theta_{S C}\right)$ as viewed from the sky to the telescope in the spherical frame of spacecraft (SC), with the azimuthal and polar angles $\varphi_{S C}$ and $\theta_{S C}$, respectively, and with the polar axis being the nominal spin axis of telescope (the center of the focal plane corresponds to $\varphi_{S C}=0^{\circ}$ and $\eta_{S C}=5^{\circ}$ ).

In this representation, the $Q$ and $U$ Stokes parameters of the beam field, being frame-dependent, are defined at each observation point with respect to the local $\varphi_{S C}, \eta_{S C}$ axes, considering $\varphi_{S C}$ (the horizontal) as the first axis (pointing to the right when viewed from the sky to the telescope) and $\eta_{\mathrm{SC}}$ as the second axis (pointing upwards). Thus, the $Q$ and $U$ are defined with respect to the parallels (horizontals) of the SC frame viewed from the sky to the telescope.

The polarization angle $\Psi_{E}$ of the beam field on the sky is measured from the positive direction of the $\varphi_{\mathrm{SC}}$ axis to the direction of major axis of polarization ellipse of the electric field $\boldsymbol{E}$, with the positive angles counted towards the positive direction of the $\eta_{S C}$ axis. So, the polarization angle is measured counter-clockwise from the parallels of the SC frame as viewed from the sky, in accordance with the definition of $Q$ and $U$. In this case, $\tan \left(2 \Psi_{E}\right)=U / Q$ that can be used for computing the polarization angle of the polarized component of partially polarized incoherent beams.

In simulations below, the nominal values of $\Psi_{E}$ for the a-channels are $\Psi_{E}=135^{\circ}$ for the beams HFI-143-1/2, 217-5/6 and 353-3/4, $\Psi_{E}=90^{\circ}$ for the beams HFI-143-3/4, 217-7/8 and 353-5/6, and $\Psi_{E}=112.5^{\circ}, 135^{\circ}, 90^{\circ}$ and $67.5^{\circ}$ for the beams HFI-100-1, 2, 3 and 4, respectively. The complementary b-channels have the PSB polarization directions in the horns precisely orthogonal to those of a-channels.

On the sky, non-orthogonality of polarization is less than $0.03^{\circ}$ for the beam-average angles $\Psi_{E}$ (evaluated using the average $Q$ and $U$ ) and, generally, less than $0.10^{\circ}$ on the beam axes (at points of maximum power), with the exception of $0.15^{\circ}$ and $0.36^{\circ}$ for the beams HFI-217-5a/b and HFI-353-3a/b, respectively. Generally, the beam-average angles $\Psi_{E}$ 
coincide with the required nominal values better than by $0.01^{\circ}$ (thus, proving the accuracy of the horn polarization directions found in [10] when using a simplified source field model), though some on-axis values may differ by $0.1^{\circ}\left(0.3^{\circ}\right.$ in the exceptional case of HFI-353-3b).

For the polarized beams, along with the distortion of polarization, some depolarization appears when the field propagates from the ideal PSB through the horn and the telescope. Similarly, for the non-polarized beams, small polarization arises. All these effects are represented by the deviations of the $Q U V$ Stokes parameters from the ideal values across the beam patterns (e.g., by non-zero values of $V$ and either $Q$ or $U$, depending on the channel, as in Fig. 2, while the ideal values are zero).

The bounds on these non-idealities are found as $\delta V=\max \left(|V| / I_{\max }\right)$ and $\delta L=\max (\delta Q, \delta U)$ where $\delta Q=\max \left(\left|Q-Q_{0}\right| / I_{\max }\right), \delta U=\max (\mid U$ $\left.-U_{0} \mid / I_{\max }\right)$ and $I_{\max }=\max (I)$, with $Q_{0}$ and $U_{0}$ being the ideal values of $Q$ and $U$, respectively (the values when the degree of polarization is either one or zero and the polarization angle $\Psi_{E}$ is equal to its nominal value across the whole beam). Notice, that the beam-average non-idealities $\delta V_{B}$ and $\delta L_{B}$ are much smaller than the maximum values $\delta V$ and $\delta L$ (typically, $\delta V_{B} \sim 0.01 \delta V$ and $\delta L_{B} \sim 0.01 \delta L$ ).

In this work, we fit the intensity Stokes parameter patterns $I\left(\varphi_{S C}, \eta_{S C}\right)$ by the elliptical Gaussian beams $F\left(\varphi_{S C}, \eta_{S C}\right)$. We normalize all the Stokes parameters so that $\max (I)=1$. In the original PO simulated beams, the power per one steradian, $P$ [Watt/sr], is computed with account of all

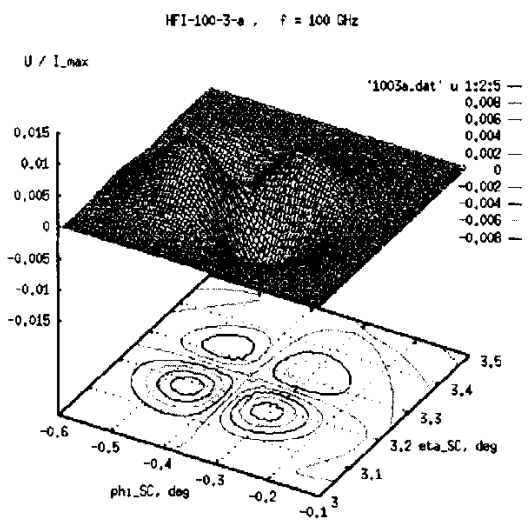

(a)

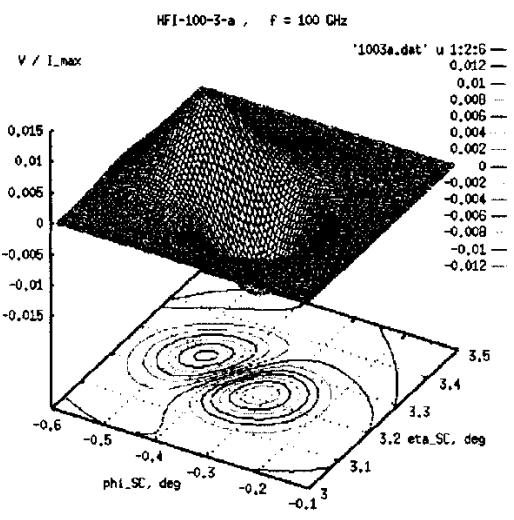

(b)

Fig. 2: The HFI-100-3a beam patterns of (a) $U$ and (b) $V$ Stokes parameters ( $\Psi_{E}=90^{\circ}$ ) 
contributing modes and polarizations, in the units of power $P_{0}$ [Watt/sr] radiated per one steradian by an isotropic source with the power of one mode of single polarization. Then, the original patterns $P\left(\varphi_{S C}, \eta_{S C}\right)$, when presented in $\mathrm{dBi}$ (Fig. 3), are computed as follows

$$
\left.P\left(\varphi_{S C}, \eta_{S C}\right)[\mathrm{dBi}]=10 \log _{10} f I\left(\varphi_{S C}, \eta_{S C}\right)\right\}+P_{\max }[\mathrm{dBi}]
$$

where $P_{\max }[\mathrm{dBi}]$ is the power gain of the original beam defined in the units of power of an isotropic source of one mode of single polarization.

In our simulations, the power of one mode constituting the unit $P_{0}$ is defined as being radiated (or received) by the bolometer of a unit crosssection $s_{0}=1 \mathrm{~mm}^{2}$ while the actual area of the bolometer $s_{B}$ is accounted in the scattering matrix $S_{n m j}$. In this case, with the incident radiation characterized by the incident field Stokes parameters $\left\{I_{s k y}, Q_{s k y}, U_{s k y}, V_{s k y}\right\}$ (in absolute units, so that $I_{s k y}[\mathrm{Watt} /(\mathrm{sr} \bullet \mathrm{Hz})]$ is the sky brightness), the total power $S_{\alpha}$ [Watt] absorbed by the bolometer of the cross-section $s_{B}$ in the given frequency band $\Delta f$ (the channel response) is evaluated as

$$
S_{\alpha}=0.5 P_{\max } \int d f \int d \Omega\left(I I_{s k y}+Q Q_{s k y}+U U_{s k y}+V V_{s k y}\right\}
$$

where the coefficient $P_{\max }$ [rel.un.] is related to the beam gain $P_{\max }[\mathrm{dBi}]$ introduced in Eq. (3) as $P_{\max }[\mathrm{dBi}]=10 \log _{10}\left(P_{\max }\right.$ [rel.un.] $)$.

For the beam fitting, we use the Gaussian function

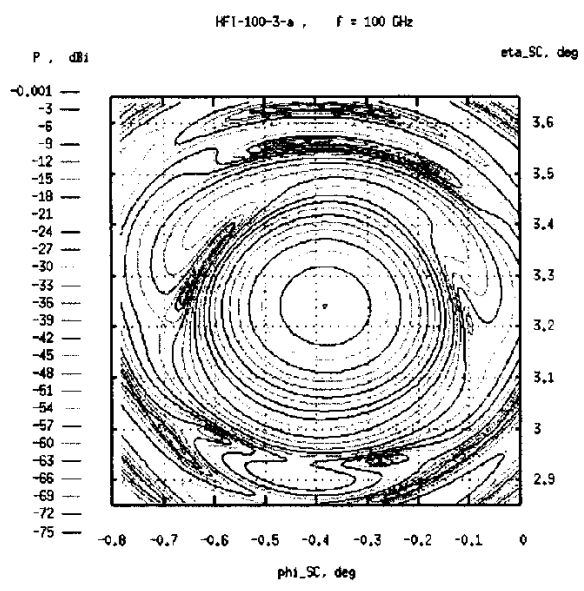

(a)

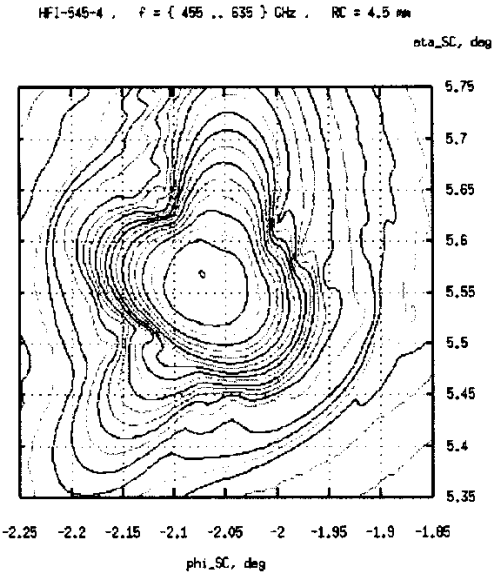

(b)

Fig. 3: The I Stokes parameter patterns of (a) HFI-100-3a and (b) HFI-545-4 beams 


$$
F\left(\varphi_{S C}, \eta_{S C}\right)=A \exp \left[-\left(p^{2} / a^{2}+t^{2} / b^{2}\right)\right]
$$

where

$$
\begin{gathered}
p=x \cos (\tau)+y \sin (\tau), \quad t=-x \sin (\tau)+y \cos (\tau), \\
x=\varphi_{S C}-\varphi_{S C O}, \quad y=\eta_{S C}-\eta_{S C}, \\
a=0.5 W_{\text {max }} / q, \quad b=0.5 W_{\min } / q, \quad q=(\ln (2))^{1 / 2}=0.8325546,
\end{gathered}
$$

$\varphi_{S C O}$ and $\eta_{S C O}$ are the angular coordinates of the center point (the point of maximum power) of the fitting elliptical Gaussian beam in the SC frame, $W_{\max }$ and $W_{\min }$ are the full beam widths at half magnitude measured along the major and minor axes of the fitting beam ellipse, respectively, and $\tau$ is the angle from the $\varphi_{S C}$ axis to the major axis of the beam ellipse measured counter-clockwise as viewed from the sky to the telescope (Fig. 4, a).

According to this definition, for each HFI beam, there are six fitting parameters $\left(W_{\max }, W_{\min }, \varphi_{S C 0}, \eta_{S C 0}, \tau\right.$, and $A$ ) which can be found by minimizing the relevant aim function that quantifies the deviation of the elliptical Gaussian fit from the given PO simulated beam. One can propose a variety of the aim functions to evaluate the difference between the fit and the actual beam. Because the difference is mainly systematic rather than random and the basic quantity of interest is the power contribution to the PSB readout, it is the deviation of the beam power

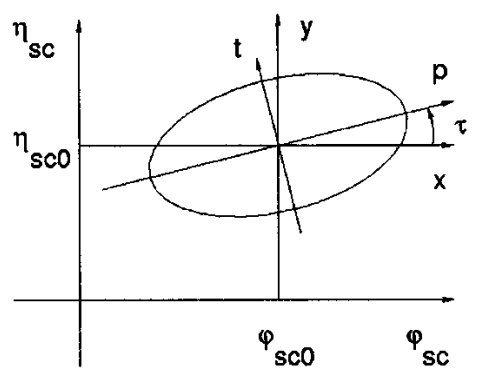

(a)

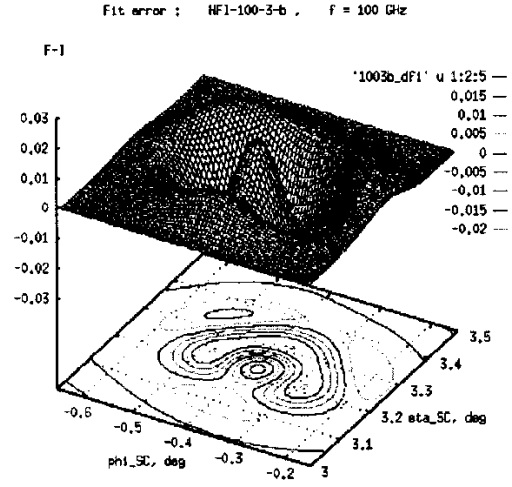

(b)

Fig. 4: (a) Parameters of the elliptical fitting beam and (b) the difference $F-I$ of power profiles of the Gaussian fit and of the original PO simulated beam HFI-100-3a 
profile from the Gaussian fit that has to be minimized. Still, even in this case, the aim function can be defined as either (i) the maximum power flux difference $\delta F=|F-I|$, or (ii) the beam-average power flux difference $\Delta F=\int \delta F d \Omega / \int I d \Omega$, or (iii) some kind of the inverse overlap integral, e.g., $D F=\left|1-\int(I F)^{1 / 2} d \Omega / \int I d \Omega\right|$ (the root mean square difference is also of interest, though it is more appropriate for random errors providing the maximum likelihood model). In addition, one may require to preserve the total power of the beam $P_{B}=\int F d \Omega=\int I d \Omega$.

\section{Results and comments}

In this work, we use the maximum power flux difference $\delta F=|F-I|$ as the aim function whose minimum provides the best Gaussian fit to the PO simulated beams under the condition of constant total power of the beam $P_{B}$. The Gaussian fitting parameters of the HFI beams obtained in this way are shown in Table 1.

Table 2 shows some other representative parameters of the HFI beams such as the full width at the $-3 \mathrm{~dB}$ level of the original PO simulated beam $\left(W_{3 d B}\right.$, see below), the mean full width at half magnitude of the Gaussian fitting beam defined as $W_{F}=\left(W_{\max } W_{\min }\right)^{1 / 2}$, the fitting beam gain $G_{F}[\mathrm{dBi}]$ $=P_{\max }[\mathrm{dBi}]+10 \log _{10}(A)$, the fitting beam ellipticity $\varepsilon_{B}=W_{\max } / W_{\min }$, the maximum fit error $\delta F$, and also the bounds $\delta L$ and $\delta V$ on the peak deviations of the $Q U V$ Stokes parameters of the original PO simulated beams from the ideal values.

Overview of the data in Tables 1 and 2 shows that the peak errors of the elliptical Gaussian fit with respect to the original beams is, typically, about $2 \%$ of maximum power for the mono-mode quasi-Gaussian beams HFI-100, 143, 217 and 353, while being around 20\% for the flat-top multi-mode beams HFI-545 and 857 . This error is quite significant, being, e.g., twice greater than the typical difference between mono-frequency and broad-band beams of polarized channels or between the beams of orthogonal polarizations of the same horns (these differences are about $1 \%$ [6]). This means that the elliptical Gaussian fit should be considered as a first term of a more advanced fitting to be developed later.

On the other hand, the fitting error remains smaller than the typical difference between the beams of two different complementary horns (the latter is about 5\% [9]). It means that the elliptical Gaussian fits are quite capable of accounting for the beam mismatch effects essential for polarization measurements. 
Table 1. Gaussian fitting parameters of the PLANCK HFI beams (multi-mode beams HFI-545 and 857 , being essentially flat-top, require extra fitting terms if the higher accuracy is needed; here, they are broadband while the other beams are computed at the central frequencies of the relevant channels)

\begin{tabular}{|c|c|c|c|c|c|c|}
\hline HFI beam & $\begin{array}{c}W_{\max } \\
{[\operatorname{arcmin}]}\end{array}$ & $\begin{array}{l}W_{\min } \\
\text { [arcmin] }\end{array}$ & $\begin{array}{c}\varphi_{S C 0} \\
{[\mathrm{deg}]}\end{array}$ & $\begin{array}{r}\eta_{S C 0} \\
{[\mathrm{deg}]}\end{array}$ & $\begin{array}{c}\tau \\
{[\mathrm{deg}]}\end{array}$ & $\begin{array}{c}A \\
\text { [rel.un.] }\end{array}$ \\
\hline HFI-100-1a & 10.3845 & 8.9658 & 1.1837 & 3.5118 & 11.2067 & 1.0176 \\
\hline HFI-100-1b & 10.5246 & 8.8356 & 1.1845 & 3.5120 & 15.0427 & 1.0201 \\
\hline HFI-100-2a & 10.3737 & 8.9862 & 0.3831 & 3.2406 & 0.6475 & 1.0109 \\
\hline HFI-100-2b & 10.3186 & 8.9803 & 0.3829 & 3.2400 & 5.9453 & 1.0171 \\
\hline HFI-100-3a & 10.2298 & 9.0679 & -0.3830 & 3.2400 & 0.0000 & 1.0152 \\
\hline HFI-100-3b & 10.3936 & 8.9468 & -0.3831 & 3.2398 & -3.0440 & 1.0143 \\
\hline HFI-100-4a & 10.3793 & 9.0000 & -1.1840 & 3.5118 & -11.6036 & 1.0144 \\
\hline HFI-100-4b & 10.5253 & 8.8361 & -1.1845 & 3.5120 & -15.0650 & 1.0201 \\
\hline HFI-14 & 7.2541 & 6.8532 & 1.3716 & 6.1966 & 48.9319 & 1.0172 \\
\hline HFI-143-1b & 7.4415 & 6.7108 & 1.3714 & 6.1964 & 46.2298 & 1.0151 \\
\hline HFI-143-2a & 7.0700 & 6.9034 & 0.5635 & 6.2243 & 68.7113 & 1.0164 \\
\hline HFI-143-2b & 7.2066 & 6.7402 & 0.5637 & 6.2250 & 56.2022 & 1.0208 \\
\hline HFI-143-3a & 7.1707 & 6.8078 & -0.5635 & 6.1991 & -68.9327 & 1.0151 \\
\hline HFI-143-3b & 7.0839 & 6.8722 & -0.5639 & 6.1994 & -38.0312 & 1.0171 \\
\hline HFI-143-4a & 7.3976 & 6.7676 & -1.4415 & 6.2212 & -56.4746 & 1.0184 \\
\hline HFI-143-4b & 7.3534 & 6.8344 & -1.4421 & 6.2208 & -38.9115 & 1.0142 \\
\hline HFI- & 7.8956 & 6.7693 & 1.1445 & 6.7339 & 60.1933 & 1.0140 \\
\hline & 7.6370 & 6.8758 & 0.2995 & 6.7606 & 81.2338 & 1.0044 \\
\hline & 7.6288 & 6.8650 & -0.2994 & 6.7358 & -78.6064 & 1.0040 \\
\hline HFI & 7.9067 & 6.8775 & -1.1438 & 6.7582 & -60.4363 & 1.0005 \\
\hline HFI-2 & 5.0197 & 4.4501 & 0.9956 & 4.0140 & 12.8744 & 1.0150 \\
\hline HFI-217-2 & 4.9764 & 4.4494 & 0.3147 & 4.0405 & 4.0827 & 1.0145 \\
\hline HFI-217-3 & 4.9977 & 4.4221 & -0.3145 & 4.0156 & -4.3018 & 1.0151 \\
\hline HFI-217-4 & 5.0628 & 4.4092 & -0.9954 & 4.0392 & -13.3840 & 1.0146 \\
\hline HFI-217-5a & 4.9833 & 4.4837 & 1.2253 & 4.5148 & 21.0541 & 1.0140 \\
\hline HFI-217-5b & 5.0052 & 4.4831 & 1.2252 & 4.5149 & 19.4575 & 1.0131 \\
\hline $\mathrm{HFI}$ & 4.9394 & 4.5089 & 0.5429 & 4.5420 & 11.4913 & 1.0123 \\
\hline$-217-6 b$ & 4.9234 & 4.5151 & 0.5433 & 4.5418 & 7.9065 & 1.0156 \\
\hline HFI-217-7a & 4.9346 & 4.5000 & -0.5433 & 4.5168 & -8.8825 & 1.0154 \\
\hline HFI-217-7b & 4.8941 & 4.5360 & -0.5431 & 4.5168 & -8.8843 & 1.0152 \\
\hline HFI-217-8a & 5.0546 & 4.4522 & -1.2249 & 4.5401 & -19.9173 & 1.0120 \\
\hline HFI-217-8b & 5.0271 & 4.4773 & -1.2249 & 4.5401 & -21.1648 & 1.0063 \\
\hline
\end{tabular}




\begin{tabular}{|l|rrrrrr|}
\hline HFI-353-1 & 5.1488 & 4.0707 & 2.0630 & 5.0155 & 20.0845 & 1.0092 \\
HFI-353-2 & 4.8530 & 4.1774 & 1.4196 & 5.0442 & 20.6846 & 1.0144 \\
HFI-353-3a & 4.8457 & 4.1255 & 0.8244 & 5.0202 & 27.5055 & 1.0199 \\
HFI-353-3b & 4.6893 & 4.2755 & 0.8246 & 5.0204 & 0.8876 & 1.0212 \\
HFI-353-4a & 4.7126 & 4.2797 & 0.2107 & 5.0461 & 27.1548 & 1.0127 \\
HFI-353-4b & 4.6685 & 4.3248 & 0.2108 & 5.0462 & -21.7530 & 1.0123 \\
HFI-353-5a & 4.7895 & 4.2136 & -0.3688 & 5.0212 & -4.8728 & 1.0119 \\
HFI-353-5b & 4.5407 & 4.4262 & -0.3687 & 5.0211 & -45.3774 & 1.0159 \\
HFI-353-6a & 4.8344 & 4.1051 & -0.9650 & 5.0454 & -14.6236 & 1.0207 \\
HFI-353-6b & 4.7200 & 4.3591 & -0.9650 & 5.0451 & -29.3738 & 1.0031 \\
HFI-353-7 & 4.8926 & 4.1895 & -1.5241 & 5.0191 & -19.7471 & 1.0055 \\
HFI-353-8 & 5.1129 & 4.0855 & -2.0631 & 5.0410 & -22.1910 & 1.0156 \\
\hline HFI-545-1 & 4.6181 & 3.8633 & 2.0715 & 5.5347 & 33.5781 & 1.1342 \\
HFI-545-2 & 4.2998 & 3.4498 & 1.4271 & 5.5666 & 30.0005 & 1.0808 \\
HFI-545-3 & 4.1421 & 3.7427 & -1.5289 & 5.5396 & -32.3890 & 1.0562 \\
HFI-545-4 & 4.5689 & 3.9371 & -2.0714 & 5.5595 & -37.8301 & 1.1296 \\
\hline HFI-857-1 & 4.5319 & 4.1268 & 0.8435 & 5.5394 & 58.3461 & 1.1252 \\
HFI-857-2 & 4.3190 & 4.2627 & 0.2289 & 5.5643 & 86.0246 & 1.1611 \\
HFI-857-3 & 4.3956 & 4.1808 & -0.3520 & 5.5399 & -71.9764 & 1.1616 \\
HFI-857-4 & 4.5436 & 4.1548 & -0.9661 & 5.5639 & -58.0198 & 1.1096 \\
\hline
\end{tabular}

Table 2. Representative characteristics of the HFI beams and their fits (beam-average non-idealities $\delta L_{B}, \delta V_{B}$ of the $Q U V$ Stokes parameters are usually about 0.01 of peak values $\delta V, \delta L$ shown in the Table)

\begin{tabular}{|c|c|c|c|c|c|c|c|}
\hline HFI beam & $\begin{array}{c}W_{3 d B} \\
\text { [arcmin] }\end{array}$ & $\begin{array}{c}W_{F} \\
\text { [arcmin] }\end{array}$ & $\begin{array}{c}G_{F} \\
{[\mathrm{dBi}]}\end{array}$ & $\varepsilon_{B}$ & $\begin{array}{l}\delta F \\
\%\end{array}$ & $\begin{array}{l}\delta L \\
\%\end{array}$ & $\begin{array}{l}\delta V \\
\%\end{array}$ \\
\hline HFI-100-1a & 9.96 & 9.65 & 60.92 & 1.158 & 1.9 & 0.8 & 2.1 \\
\hline HFI-100-1b & 9.93 & 9.64 & 60.92 & 1.191 & 1.9 & 0.6 & 2.0 \\
\hline HFI-100-2a & 9.93 & 9.66 & 60.91 & 1.155 & 1.9 & 0.9 & 1.4 \\
\hline HFI-100-2b & 9.92 & 9.63 & 60.94 & 1.151 & 2.0 & 0.8 & 1.3 \\
\hline HFI-100-3a & 9.93 & 9.63 & 60.94 & 1.128 & 2.3 & 0.9 & 1.4 \\
\hline HFI-100-3b & 9.89 & 9.64 & 60.93 & 1.162 & 2.0 & 0.7 & 1.3 \\
\hline HFI-100-4a & 9.96 & 9.67 & 60.90 & 1.153 & 1.9 & 0.8 & 2.1 \\
\hline HFI-100-4b & 9.93 & 9.64 & 60.92 & 1.191 & 1.9 & 0.6 & 2.0 \\
\hline HFI-143-1a & 7.24 & 7.05 & 63.53 & 1.058 & 1.9 & 1.6 & 4.0 \\
\hline HFI-143-1b & 7.19 & 7.07 & 63.52 & 1.109 & 1.8 & 1.5 & 4.1 \\
\hline HFI-143-2a & 7.14 & 6.99 & 63.64 & 1.024 & 1.9 & 1.2 & 3.7 \\
\hline HFI-143-2b & 7.15 & 6.97 & 63.65 & 1.069 & 2.2 & 1.1 & 3.7 \\
\hline
\end{tabular}




\begin{tabular}{|c|c|c|c|c|c|c|c|}
\hline HFI-143-3a & 7.12 & 6.99 & 63.63 & 1.053 & 1.8 & 1.3 & 3.7 \\
\hline HFI-143-3b & 7.13 & 6.98 & 63.64 & 1.031 & 1.9 & 1.1 & 3.7 \\
\hline HFI-143-4a & 7.24 & 7.08 & 63.50 & 1.093 & 2.0 & 1.7 & 4.6 \\
\hline HFI-143-4b & 7.20 & 7.09 & 63.48 & 1.076 & 1.9 & 1.5 & 4.5 \\
\hline HFI-143-5 & 7.36 & 7.31 & 63.24 & 1.166 & 2.1 & 1.0 & 4.7 \\
\hline HFI-143-6 & 7.29 & 7.25 & 63.33 & 1.111 & 1.8 & 0.9 & 4.4 \\
\hline HFI-143-7 & 7.26 & 7.24 & 63.34 & 1.111 & 1.9 & 0.9 & 4.3 \\
\hline HFI-143-8 & 7.37 & 7.37 & 63.16 & 1.150 & 2.0 & 1.0 & 4.7 \\
\hline HFI-217-1 & 4.82 & 4.73 & 67.10 & 1.128 & 1.9 & 0.7 & 1.5 \\
\hline HFI-217-2 & 4.81 & 4.71 & 67.15 & 1.118 & 1.7 & 0.7 & 0.5 \\
\hline HFI-217-3 & 4.79 & 4.70 & 67.16 & 1.130 & 1.7 & 0.7 & 0.5 \\
\hline HFI-217-4 & 4.84 & 4.72 & 67.10 & 1.148 & 1.9 & 0.7 & 1.5 \\
\hline HFI-217-5a & 4.83 & 4.73 & 67.10 & 1.111 & 2.0 & 0.4 & 2.2 \\
\hline HFI-217-5b & 4.83 & 4.74 & 67.09 & 1.116 & 2.1 & 0.6 & 2.3 \\
\hline HFI-217-6a & 4.81 & 4.72 & 67.14 & 1.095 & 2.0 & 0.4 & 1.3 \\
\hline HFI-217-6b & 4.80 & 4.72 & 67.15 & 1.090 & 2.0 & 0.4 & 1.4 \\
\hline HFI-217-7a & 4.81 & 4.72 & 67.16 & 1.097 & 2.0 & 0.3 & 1.6 \\
\hline HFI-217-7b & 4.80 & 4.72 & 67.16 & 1.079 & 2.0 & 0.5 & 1.5 \\
\hline HFI-217-8a & 4.83 & 4.74 & 67.07 & 1.135 & 2.2 & 0.4 & 2.5 \\
\hline HFI-217-8b & 4.82 & 4.74 & 67.08 & 1.123 & 2.2 & 0.7 & 2.3 \\
\hline HFI-353-1 & 4.64 & 4.58 & 67.63 & 1.265 & 2.6 & 3.5 & 2.4 \\
\hline HFI-353-2 & 4.62 & 4.50 & 67.81 & 1.162 & 2.4 & 2.7 & 1.8 \\
\hline HFI-353-3a & 4.60 & 4.47 & 67.90 & 1.175 & 1.9 & 2.8 & 3.6 \\
\hline HFI-353-3b & 4.60 & 4.48 & 67.88 & 1.097 & 2.0 & 2.8 & 4.2 \\
\hline HFI-353-4a & 4.60 & 4.49 & 67.86 & 1.101 & 1.4 & 2.8 & 3.8 \\
\hline HFI-353-4b & 4.60 & 4.49 & 67.85 & 1.079 & 1.5 & 2.8 & 3.9 \\
\hline HFI-353-5a & 4.59 & 4.49 & 67.86 & 1.137 & 1.4 & 2.7 & 4.1 \\
\hline HFI-353-5b & 4.60 & 4.48 & 67.88 & 1.026 & 1.6 & 2.8 & 3.4 \\
\hline HFI-353-6a & 4.57 & 4.45 & 67.92 & 1.178 & 2.0 & 2.9 & 4.2 \\
\hline HFI-353-6b & 4.65 & 4.54 & 67.76 & 1.083 & 2.2 & 2.8 & 3.9 \\
\hline HFI-353-7 & 4.62 & 4.53 & 67.76 & 1.168 & 2.8 & 2.8 & 1.9 \\
\hline HFI-353-8 & 4.65 & 4.57 & 67.66 & 1.251 & 2.6 & 3.5 & 2.4 \\
\hline HFI-545-1 & 4.87 & 4.22 & 76.58 & 1.195 & 14.8 & 2.5 & 7.5 \\
\hline HFI-545-2 & 4.58 & 3.85 & 77.33 & 1.246 & 27.7 & 2.9 & 6.6 \\
\hline HFI-545-3 & 4.57 & 3.94 & 77.14 & 1.107 & 23.2 & 2.7 & 7.0 \\
\hline HFI-545-4 & 4.88 & 4.24 & 76.54 & 1.160 & 14.3 & 2.5 & 7.5 \\
\hline HFI-857-1 & 5.15 & 4.32 & 79.75 & 1.098 & 21.2 & 7.0 & 2.4 \\
\hline HFI-857-2 & 5.17 & 4.29 & 79.84 & 1.013 & 22.5 & 7.4 & 1.8 \\
\hline HFI-857-3 & 5.17 & 4.29 & 79.85 & 1.051 & 22.3 & 6.7 & 1.8 \\
\hline HFI-857-4 & 5.15 & 4.34 & 79.70 & 1.094 & 20.7 & 6.9 & 2.6 \\
\hline
\end{tabular}


Another essential observation is that the beam width of the Gaussian fit $\left(W_{F}\right)$ is noticeably smaller than the typical width of the original beam evaluated in one or another manner, e.g., as a diameter of the circle of the same area as bounded by the $-3 \mathrm{~dB}$ isolevel $\left(W_{3 d B}\right)$ or by the isolevel comprising $50 \%$ of the total power of the beam $\left(W_{50}\right)$.

The reason is that the real beams have specific shape at the top, tending to be flat-top, though more complicated. This happens even to quasiGaussian beams (see Fig. 4, b), both due to the field propagation via the horns and via the telescope, though multi-mode beams are significantly more flat-top because of their modal composition. As a result, the original beams at half magnitude are always wider compared to the Gaussian fits at their half magnitude, respectively. More complicated beam fitting, when using additional terms, would be able to represent the width of the original beams with a better accuracy.

\section{Conclusions}

We computed polarized mono-mode and non-polarized multi-mode HFI beams of the ESA PLANCK telescope by means of the physical optics propagation of the horn aperture field simulated by the scattering matrix approach. Beam patterns of Stokes parameters have been evaluated and the required polarization angles on the horn apertures obtained. The patterns have been studied for the estimates of systematic errors in polarization measurements.

Computed power patterns are fitted by the elliptical Gaussian beams minimizing the peak difference between the power distributions of the Gaussian fit and the actual beam under the condition of constant total power. The fitting error is, typically, about $2 \%$ of maximum power for the mono-mode quasi-Gaussian beams and about $20 \%$ for the flat-top multimode beams. Basic elliptical Gaussian fitting is capable of representing the main differences between the beams of different channels, although more complicated fitting is needed when the higher accuracy is required.

\section{Acknowledgement}

This work was supported by the Enterprise Ireland Basic Research Grant and the French-Irish Ulysses Research Visit Grants 2002 and 2003.

The authors are grateful to B. Maffei for the horn design, to E. Gleeson for the scattering matrix coefficients of the horn fields, and to Y. Longval 
for providing the positions of the telescope focal points and orientations of the horn axes.

\section{References}

1. J. A. Tauber, "The PLANCK mission: overview and current status," Astrophys. Lett. Comm., vol. 37, pp. 145-150 (2000)

2. J.-M. Lamarre et al., "The Planck High Frequency Instrument, a third generation CMB experiment, and a full sky submillimeter survey," New Astronomy Reviews, vol. 47, no. 11-12, p. 1017-1024 (2003).

3. B. Maffei, P. A. R. Ade, C. E. Tucker, E. Wakui, R. J. Wylde, J. A. Murphy, and R. M. Colgan, "Shaped corrugated horns for cosmic microwave background anisotropy measurements", Int. J. Infrared and Millimetre Waves, vol. 21, no. 12, p. 2023-2033, (2000)

4. A. D. Turner et al., "Silicon nitride micromesh bolometer array for submillimeter astrophysics," Applied Optics, vol. 40, pp. 4921-4932 (2001)

5. P. Fosalba, O. Doré, and F. R. Bouchet, "Elliptical beams in CMB temperature and polarization anisotropy experiments: An analytic approach, Phys. Rev.D, vol. 65, 063003 (2002)

6. V. B. Yurchenko, J. A. Murphy, and J.-M. Lamarre, "Fast physical optics simulations of the multi-beam dual-reflector submillimeterwave telescope on the ESA PLANCK Surveyor," Int. J. Infrared and Millimeter Waves, vol. 22, no. 1. p. 173-184 (2001)

7. V. B. Yurchenko, J. A. Murphy, and J.-M. Lamarre, "PLANCK HFI beam simulations for polarized and multi-moded horns," $25^{\text {th }}$ ESA Antenna Workshop on Satellite Antenna Technology, 18-20 September, 2002, ESTEC, Noordwijk, The Netherlands. K. van 't Klooster and L. Fanchi, Eds, pp. 281-286 (2002)

8. E. Gleeson, J. A. Murphy, B. Maffei, J.-M. Lamarre, and R. J. Wylde, "Definition of the multi-mode horns for the HFI instrument on PLANCK," ibid., pp. 649-655 (2002)

9. V. B. Yurchenko, J. A. Murphy, and J.-M. Lamarre, "Simulation and comparison of the PLANCK HFI beams with implications on polarization measurements," $3 r d$ ESA Workshop on Millimetre Wave Technology and Applications, May 21-23, 2003, MilliLab, Espoo, 
Finland. J. Mallat, A. Raisanen and J. Tuovinen, Eds, pp. 187-192 (2003)

10. V. B. Yurchenko, "Measuring CMB polarization with ESA PLANCK submm-wave telescope," Experimental Cosmology at Millimetre Wavelengths: 2K1BC Workshop, Breuil-Cervinia, Italy, July 9-12, 2001. M. De Petris, P. A. Moro, and M. Gervasi, Eds. AIP Conf. Proc., vol. 616. pp. 234-238 (2002) 